\title{
Die Entwicklung bayerischer Hochschulrechenzentren - Handlungsempfehlungen aus einer Fallstudie für ein professionelles IT-Servicemanagement
}

\author{
Claudius Jonas $\mathbb{D} \cdot$ Jonathan Lautenschlager $\mathbb{D} \cdot$ Torsten Eymann $\mathbb{D}$
}

Eingegangen: 29. März 2021 / Angenommen: 8. Juli 2021 / Online publiziert: 2. August 2021

(C) Der/die Autor(en) 2021, korrigierte Publikation 2021

Zusammenfassung Im digitalen Informationszeitalter stellen Hochschulrechenzentren (HRZ) einen zentralen Bestandteil zur Erfüllung operativer Aufgaben in Forschung, Lehre und Verwaltung von Hochschulen dar. Dabei stehen HRZ vor unterschiedlichen Herausforderungen, die durch diverse Nutzeranforderungen sowie Anforderungen bezüglich IT-Sicherheit und Regulatorik hervorgerufen werden. Um diese Herausforderungen zu bewältigen, ist es essenziell, bisher dezentral und heterogen geprägte IT-Services stetig zu professionalisieren. Dadurch entsteht die Notwendigkeit, sich für einen nachhaltigen Betrieb der HRZ stärker an etablierten Servicestandards und Anforderungen von hochschulinternen sowie hochschulexternen Nutzergruppen zu orientieren. Ein professionelles IT-Servicemanagement (ITSM) wird damit zur Grundlage für HRZ. Im Rahmen eines durch das bayerische Wissenschaftsministerium geförderten Projektes wird durch eine vergleichende Fallstudie zum einen der Status des ITSM der bayerischen HRZ systematisch erfasst, zum anderen darauf aufbauend eine Weiterentwicklung unterstützt. Dazu wurden Interviews und Selbstevaluierungen von neun unterschiedlichen Hochschulen durchgeführt und die Ergebnisse anschließend evaluiert. In diesem Beitrag werden die zentralen Erkenntnisse vorgestellt und Handlungsempfehlungen daraus abgeleitet.

Schlüsselwörter IT-Servicemanagement · Hochschulrechenzentren · Fallstudie · Handlungsempfehlungen

Claudius Jonas $(\bowtie) \cdot$ Jonathan Lautenschlager $\cdot$ Torsten Eymann

Projektgruppe Wirtschaftsinformatik des Fraunhofer FIT, Kernkompetenzzentrum Finanz- \& Informationsmanagement, Universität Bayreuth, Universitätsstraße 30, 95447 Bayreuth, Deutschland E-Mail: claudius.jonas@uni-bayreuth.de 


\title{
The Development of Bavarian University Computer Centres - Recommendations for Action from a Case Study for Professional IT Service Management
}

\begin{abstract}
In the digital information age, university computer centers (UCCs) are a central component for fulfilling operational tasks in research, teaching, and administration at universities. In doing so, UCCs face various challenges caused by very diverse user requirements and requirements regarding IT security and regulation. It is essential to continuously professionalize IT services that were previously decentralized and heterogeneous to meet these challenges. This professionalization makes it necessary to focus the UCC's long-term operation more strongly on established service standards and internal and external university user groups' requirements. Thus, professional IT service management (ITSM) becomes necessary for UCC. As part of a project funded by the Bavarian Ministry of Science, we conduct a comparative case study to systematically assess the status of ITSM in Bavarian UCCs and support further development. For this purpose, interviews and self-evaluations of nine different universities were conducted and subsequently evaluated. Thus, this paper presents the central findings and recommendations for UCCs.
\end{abstract}

Keywords IT service management - University computer centers · Case study · Recommendations for action

\section{Motivation}

Im heutigen digitalen Informationszeitalter sind die Hochschulrechenzentren (HRZ) eine wesentliche Säule zur Erfüllung der Aufgaben in Forschung, Lehre und Verwaltung und bilden einen zentralen Knotenpunkt innerhalb der jeweiligen Hochschule, aber auch über Hochschulgrenzen hinweg. Dabei stellt eine generell steigende Erwartungshaltung an die technische Infrastruktur, beispielsweise durch immer mehr WLAN-fähige Endgeräte, durch spezifische Anforderungen unterschiedlicher Nutzer, aber auch neue oder geänderte Nutzungsszenarien, die beispielweise durch stärkere Online-Kollaborationen entstehen, große Herausforderungen für HRZ dar. Zusätzlich spielen auch regulatorische Anforderungen, zum Beispiel die DSGVO ${ }^{1}$ und das $\mathrm{EGovG}^{2}$, und die Gewährleistung von IT-Sicherheit und Verfügbarkeit eine immer wichtigere Rolle. Speziell durch die Zunahme von IT-Sicherheitsvorfällen oder auch Cyberattacken hat das Thema IT-Sicherheit große Aufmerksamkeit in Deutschland (Abolhassan 2017; Zwilling et al. 2020) und dadurch auch im Kontext der über 424 registrierten Hochschulen erhalten (Rudnicka 2020). In diesem Zusammenhang ist in der Hochschulrektorenkonferenz auch das Thema Informationssicherheit als ein wichtiger Bestandteil der Aufgaben der Hochschulleitung benannt worden, welches nicht nur Themen der IT-Sicherheit umfasst, sondern konkret noch darüber hinaus gehen soll, dadurch dass auch informationsverarbeitende Prozesse

\footnotetext{
1 DSGVO steht für Datenschutz-Grundverordnung, siehe www.dsgvo-gesetz.de.

2 EGovG steht für E-Government-Gesetz, siehe www.gesetze-im-internet.de/egovg.
} 
in den Vordergrund von Risikobewertung und -behandlung gestellt werden sollen (Hochschulrektorenkonferenz 2018).

Aufgrund dessen werden nicht nur die internen Anforderungen an die IT-Systemlandschaft, sondern auch der Anforderungsdruck von außen, immer komplexer (Bick und Börgmann 2008). Beispielsweise zeigen die Auswirkungen der CoronaPandemie und dem damit verbundenen Digitalisierungsdruck, wie reaktionsschnell und agil Hochschulen auf veränderte äußere Bedingungen reagieren müssen. Dies hat zur Folge, dass verstärkt alternative digitale Lehrangebote zum Einsatz kommen, sodass innerhalb kürzester Zeit neue Software und IT-Infrastruktur zur Verfügung gestellt werden muss, um auf diese Veränderungen flexibel reagieren zu können (Williamson et al. 2020). Obwohl die, durch die Corona-Pandemie induzierten, Anpassungen von IT-Services in Studium und Lehre insgesamt als positiv bewertet werden (Lörz et al. 2020), fehlt es den Hochschulen und HRZ an Strategien, die kürzlich eingeführten IT-Services zu verstetigen und langfristig in das Serviceportfolio aufzunehmen (Bosse et al. 2020). Doch auch unabhängig von solchen disruptiven gesamtgesellschaftlichen Einflüssen müssen sich HRZ stetig professionalisieren und von der bloßen Bereitstellung von IT hin zu professionellen Anbietern von ITServices entwickeln, um den sich kontinuierlich verändernden Anforderungen und unterschiedlichen Nutzern in der Zukunft gerecht zu werden. Dementsprechend entsteht die Notwendigkeit, sich für einen zukunftsorientierten und richtungsweisenden Betrieb der bayerischen HRZ stärker an etablierten Servicestandards, Anforderungen von hochschulinternen Nutzern sowie hochschulexternen Potenzialen zu orientieren. Ein professionelles IT-Servicemanagement (ITSM) wird damit nicht nur in der Industrie zur essenziellen Grundlage, sondern auch für Hochschulen und deren Rechenzentren.

In diesem Zusammenhang ist es für die Dienstleister und somit auch für die Hochschulen nicht mehr ausreichend, sich ausschließlich auf die Technologie und ihre internen Prozesse zu konzentrieren. Vielmehr sind diese dazu gezwungen, auch die Qualität, der von ihnen erbrachten Dienstleistungen zu berücksichtigen und den Anforderungen an Forschung und Lehre gerecht zu werden. Unternehmen und Dienstleister orientieren sich dazu an einschlägigen Referenzmodellen, wie zum Beispiel ITIL $^{\circledR}$, oder Servicestandards, wie beispielsweise ISO/IEC 20000, um die eigenen IT-Services resilient und robust gegenüber den zunehmenden externen Anforderungen bereitzustellen (Bon 2008). Bick und Börgmann (2008) untersuchten den Einsatz von ITSM an deutschen Hochschulen und stellten unter anderem fest, dass es keinen gesamtheitlichen Ansatz zum Thema ITSM gibt, sich aber ein Großteil der beteiligten Hochschulen der Thematik angenommen haben, wobei es sich hier insbesondere um individuelle Herangehensweisen der einzelnen HRZ zur Umsetzung des hochschuleigenen ITSM handelt. Im Kontext der IT-Governance an HRZ untersuchten Hotzel et al. (2015) die Rolle des Chief Information Officers (CIO) an deutschen HRZ und kamen unter anderem zu dem Ergebnis, dass über die Hälfte der beteiligten HRZ keinen CIO beziehungsweise einen CIO, der auch Teil der Hochschulleitung ist, besitzen. An deutschen HRZ werden zwar erste Ansätze der IT-Governance und IT-Sicherheit, beziehungsweise Informationssicherheit, diskutiert, jedoch lässt sich darüber hinaus kein einheitliches Verständnis zum Thema ITSM feststellen. Diese Problemstellung adressiert das vom Bayerischen Staatsministerium für Wissen- 
schaft und Kunst (StMWK) geförderte Forschungsprojekt „IT-Servicemanagement für bayerische Hochschulrechenzentren “ als Teil des Programms „Digitaler Campus Bayern". Ziel des Projektes ist es zum einen durch eine vergleichende Fallstudie den Status des ITSM der HRZ im bayerischen Raum systematisch zu erfassen und zum anderen diese bei der Verbesserung und Weiterentwicklung zu unterstützen. Im Zuge dessen beantworten wir auf Basis unserer Forschungsaktivitäten in diesem Beitrag die folgende Forschungsfrage:

Welche Erkenntnisse und Handlungsempfehlungen lassen sich für das ITSM

bayerischer HRZ auf Basis des aktuellen Status Quo ableiten?

Dazu wurde im ersten Schritt unter Berücksichtigung geeigneter Standards, Zertifizierungen und Normen ein geeignetes Evaluierungsinstrument adaptiert und auf dessen Basis ein hochschulspezifischer Analyserahmen zur Erfassung des ITSM erstellt. Mit Hilfe des Analyserahmens wurden Interviews und Evaluierungen mit insgesamt neun Hochschulen innerhalb Bayerns durchgeführt. Anschließend wurden die Ergebnisse der Erhebung bewertet und in Bezug zu gesetzlichen Vorgaben und hochschulpolitischen Zielen gesetzt. Im vierten Schritt wurden auf Basis der Interviews und Auswertungen der Erhebungen Handlungsempfehlungen konzipiert, die speziell auf unterschiedliche Stufen hin zur Etablierung eines gemeinsamen Ansatzes eines „Shared Service Centers“3 ${ }^{\text {" }}$ ugeschnitten sind. Letztlich sollen diese Ergebnisse genutzt werden, um in individuellen Workshops Umsetzungsempfehlungen für entsprechende HRZ zu vermitteln und somit Hochschulen bei der Entwicklung des hochschulspezifischen ITSM zu unterstützen.

\section{IT-Servicemanagement in bayerischen Hochschulrechenzentren}

Insbesondere die Etablierung eines hochschulspezifischen ITSM stellt HRZ weltweit vor infrastrukturelle und auch organisatorische Herausforderungen (Zhen und Xin-yu 2007; Iden und Eikebrokk 2013). Dabei lässt sich unter dem Begriff ITSM ein Ansatz für den IT-Betrieb von Service-Dienstleistern verstehen, der sich dadurch auszeichnet, dass er den Schwerpunkt auf IT-Services, Kunden, Service Level Agreements und die Abwicklung der täglichen Aktivitäten mittels IT-Servicefunktionen legt (Iden und Eikebrokk 2013). Im Zuge dessen ist ein Ansatz, diese Herausforderungen zu adressieren und die eigenen hochschulspezifischen Services der Universitätsmitarbeitenden und Studierenden zu professionalisieren. Konkret bedeutet dies, die Qualität und ganzheitliche Verfügbarkeit der einzelnen Services für Mitarbeitende und Studierende der Hochschule sowie für Dritte zu verbessern und aufzuwerten. Ferner soll durch die Einführung eines professionellen ITSM eine trennscharfe Entwicklung des eigenen Serviceportfolios erarbeitet werden (Fähnrich und Bogdan 2010).

Diese Professionalisierung wird mittels einer Zertifizierung von steuerungsspezifischen Anforderungen des ITSM umgesetzt und in Form etablierter Standards und

\footnotetext{
3 Shared Service Center heißt, dass anstelle der bloßen Bereitstellung von IT verstärkt Dienstleistungen beziehungsweise kundenorientierte Services angeboten werden.
} 
Rahmenwerke vereinheitlichend konsolidiert. Zur geeigneten Auswahl wurden daher im Rahmen des ITSM-Projekts bekannte Rahmenwerke wie Cobit ${ }^{\circledR}$ oder ITIL ${ }^{\circledR}$ betrachtet. Dabei ordnet sich das ITSM zwischen den drei übergeordneten Kategorien, den Rahmenwerken des Qualitätsmanagements (z.B. ISO 9000), den Rahmenwerken der Informationssicherheit (z. B. ISO/IEC 27000, ISIS12 ${ }^{4}$ und BSI ITGrundschutz ${ }^{5}$ ) und den Rahmenwerken der Softwareentwicklung (z. B. ISO 15504), ein (Rohrer und Söllner 2017). Diese Rahmenwerke bedienen inhaltlich sowohl IT-sicherheitsspezifische als auch regulatorische Anforderungen, unterscheiden sich jedoch in ihrer inhaltlichen Ausrichtung voneinander. Im Rahmen dieses Beitrags und des zu Grunde liegenden Projekts steht insbesondere ITSM im Fokus. Cobit ${ }^{\circledR}$ oder ITIL ${ }^{\circledR}$ befassen sich im Zuge dessen ganzheitlich und sehr detailliert mit der inhaltlichen Implementierung von ITSM vor allem in Konzernen oder Großunternehmen (Bon 2008; Gaulke 2019; Beims und Ziegenbein 2021). Dieser Detailgrad übersteigt jedoch oftmals die Kapazitäten und die Zielsetzung für vergleichsweise kleinere HRZ, sodass eine Adaption dieser zum Teil komplexen Rahmenwerke die HRZ vor große Herausforderungen stellt.

Das Rahmenwerk FitSM basiert in diesem Zusammenhang auf verschiedenen Rahmenwerken zum Thema ITSM und bietet eine vergleichsweise leichtgewichtigere Alternative zu den etablierten Rahmenwerken Cobit ${ }^{\circledR}$ oder ITIL ${ }^{\circledR}$ (Rohrer und Söllner 2017). Ziel von FitSM ist es, einen einfachen, verständlichen und praktisch zu nutzenden Ansatz für das ITSM zu schaffen, speziell entwickelt für kleinund mittelständische Unternehmen (FitSM 2016). Übergeordnet bedient sich FitSM dreier Rahmenwerke und Standards (Cobit ${ }^{\circledR}$, ITIL ${ }^{\circledR}$ und der ISO/IEC 20000 Norm). Die internationale Norm ISO/IEC 20000 beschreibt Mindestanforderungen für ein geregeltes ITSM, nach der sich Organisationen zertifizieren lassen können, während Cobit ${ }^{\circledR}$ seinen Fokus auf die IT-Governance legt und ITIL ${ }^{\circledR}$ die ITSM-spezifischen Themeninhalte adressiert (Rohrer und Söllner 2017). Gleichzeitig bedient sich FitSM aus den Bereichen des Qualitätsmanagements (ISO 9000), den Standards der Informationssicherheit (ISO/IEC 27000, ISIS12 und BSI IT-Grundschutz) sowie den Standards für Reifegrad- und Softwareentwicklung (ISO 15504). Das Zusammenspiel und die Vernetzung dieser Rahmenwerke ist in FitSM gebündelt, welches sich dadurch sowohl als ISO/IEC 20000 konforme als auch als leicht verständliche und pragmatische Option zur Implementierung in bayerischen HRZ anbietet (Tiemeyer 2020).

Neben der architektonischen Zusammensetzung gliedert sich das Rahmenwerk FitSM inhaltlich in sieben grundlegende Anforderungen (GR 1-7) und 14 spezifische Anforderungen (PR 1-14), welche in abgewandelter Form auch in der ISO/IEC 20000 Norm und in den ITIL ${ }^{\circledR}$-Rahmenwerken wiederzufinden sind. Während die grundlegenden Anforderungen vor allem die Rahmenbedingungen für eine kontinuierliche Entwicklung des generellen ITSM bedienen (z.B. Verpflichtung des Top-

\footnotetext{
4 ISIS12 steht für Informationssicherheitsmanagementsystem in 12 Schritten, siehe isis12.it-sicherheitsclu ster.de.

5 BSI IT-Grundschutz steht für Bundesamt für Sicherheit in der Informationstechnik IT-Grundschutz, siehe www.bsi.bund.de/DE/Themen/Unternehmen-und-Organisationen/Standards-und-Zertifizierung/ITGrundschutz/it-grundschutz_node.html.
} 


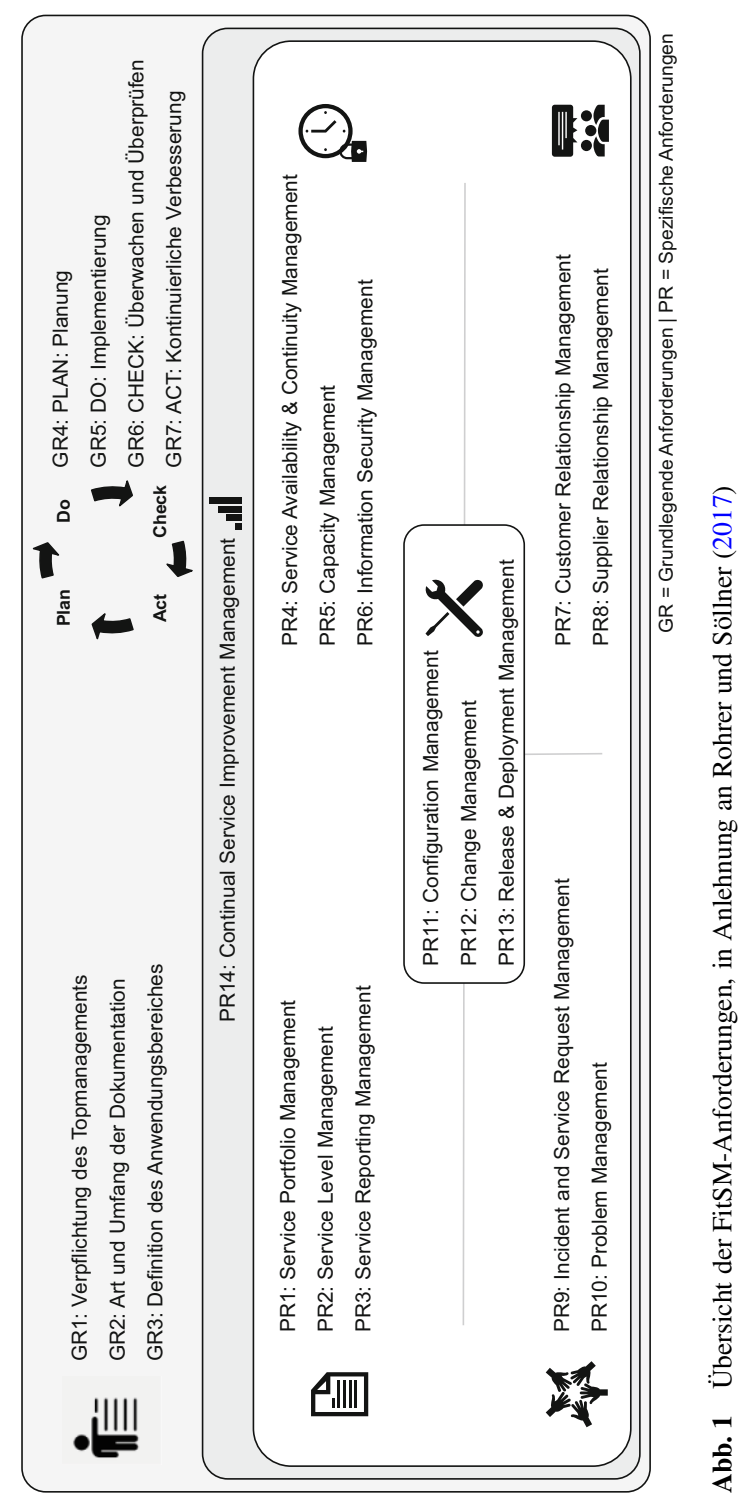


Managements, Art und Weise der Dokumentation oder die fortlaufende Entwicklung des ITSM durch einen iterativen PDCA-Zyklus ${ }^{6}$ ), erstreckt sich das inhaltliche Profil der spezifischen Anforderungen über die unterschiedlichen Bereiche des ServicePortfolios (z. B. Service Portfolio Management, Information Security Management oder Customer Relationship Management). Abb. 1 gibt einen Überblick über die leichtgewichtigen Anforderungen des FitSM-Rahmenwerks an das ITSM bayerischer HRZ (Rohrer und Söllner 2017).

Die zunehmende Digitalisierung und die damit einhergehenden diversen Nutzer-, regulatorischen und IT-sicherheitsspezifischen Anforderungen setzen die HRZ insbesondere durch limitierte Ressourcen und fehlende IT-Infrastrukturen erheblich unter Druck. Dieser zunehmende Druck spiegelt sich auch in der breiten inhaltlichen Ausrichtung der verschiedenen grundlegenden und spezifischen Anforderungen des FitSM-Rahmenwerkes wider. Dabei sind HRZ einerseits dazu gezwungen, den unterschiedlichen Anforderungen in der Forschung und Lehre der verschiedenen Lehrstühle gerecht zu werden, andererseits stehen diese aber auch vor der Herausforderung, die zunehmende dezentrale und heterogene Prägung der IT-Infrastruktur der Hochschule zu gewährleisten.

Folgerichtig hat das Wachstum und die Professionalisierung der IT-Infrastruktur von Institutionen des öffentlichen Sektors, unter anderem für HRZ, stetig an Bedeutung gewonnen. Gerade in Bezug auf die zunehmende Nachfrage nach Lösungen für einen digitalen Universitätsbetrieb - insbesondere in Zeiten der Corona-Pandemie erscheint es sinnvoll, die Entwicklung entsprechender Handlungsfelder und Umsetzungsempfehlungen für HRZ bereitzustellen, die dafür notwendig sind, das ITSM dieser Hochschulen zu professionalisieren und die Herausforderungen der Digitalisierung bezüglich des Bereitstellens infrastruktureller Kapazitäten zu fördern.

\section{Projektvorgehen}

Das Entwickeln und Ableiten von Handlungsempfehlungen für ITSM an HRZ gliedert sich dabei in vier Phasen (Abb. 2).

In Phase 1 bestand das Ziel ein Evaluierungsinstrument zur Erhebung des Status Quo des ITSM an HRZ zu entwickeln. Hierfür erfolgte eine Analyse und ein Vergleich etablierter Rahmenwerke des ITSM. Aus der Konsolidierung dieser Rahmenwerke wurde ein Analysetool entwickelt, das basierend auf sieben grundlegende

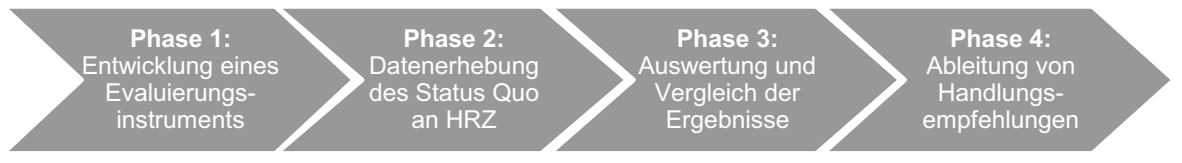

Abb. 2 Gesamtüberblick über das Projektvorgehen

\footnotetext{
6 Der PDCA-Zyklus ist eine methodische Vorgehensweise mit dem Ziel, den Umfang, Detailgrad sowie auch die Anforderungen an ein ITSM zu planen, diese umzusetzen und nachhaltig weiterzuentwickeln (Rohrer und Söllner 2017).
} 


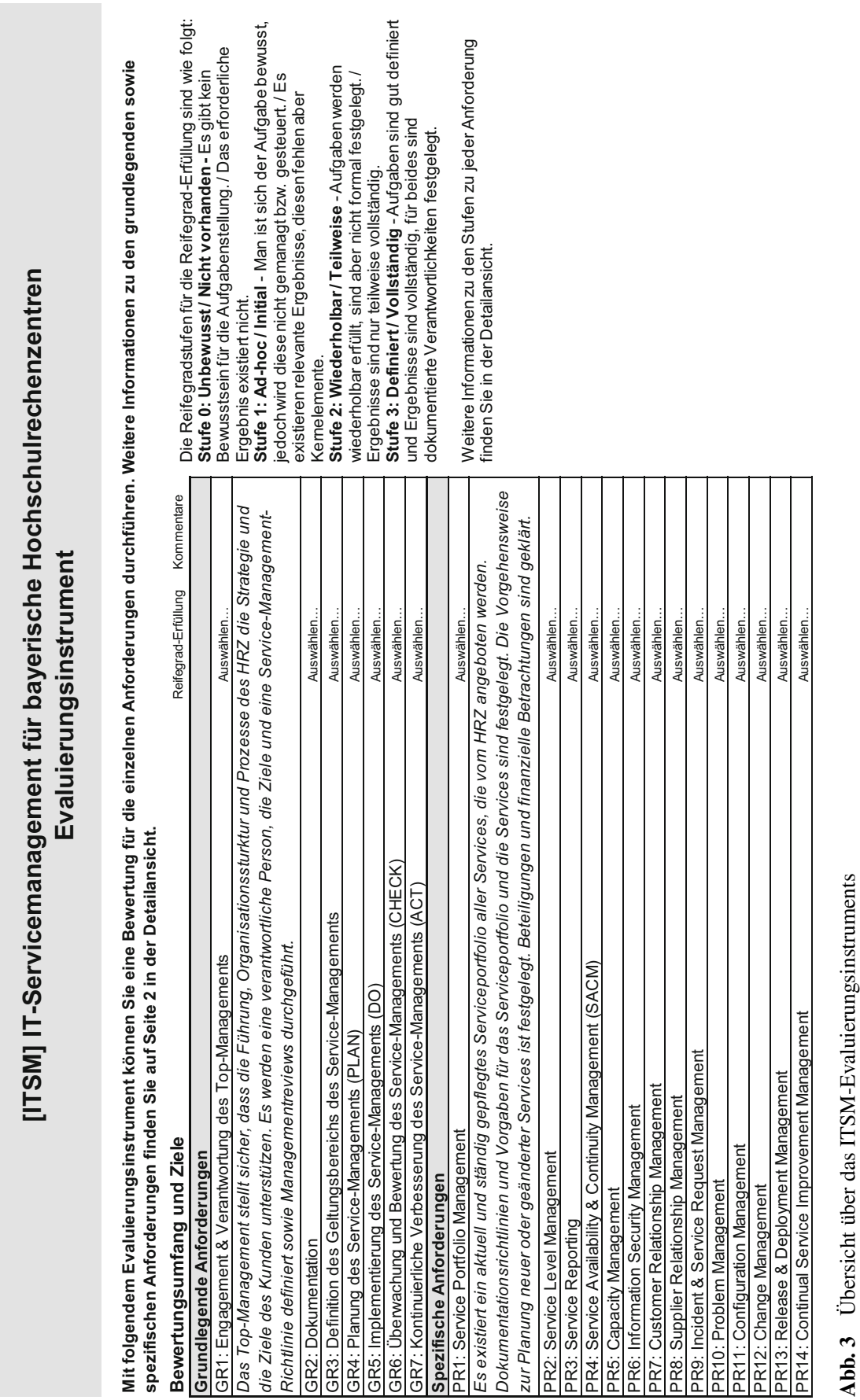


Anforderungen, die sich auf die notwendige Basis zu einem erfolgreichen Betrieb des ITSM richten, sowie 14 spezifische Anforderungen (Abb. 1), den Status Quo des ITSM der jeweiligen HRZ erheben sollte (Rohrer und Söllner 2017). Zur Analyse und zur Bewertung des erhobenen Status Quo haben wir auf Basis dieser sieben grundlegenden und 14 spezifischen Anforderungen ein trennscharfes Reifegradprofil festgelegt. Dieses Anforderungsprofil wurde in Form eines Evaluierungsinstruments zusammengefasst und um Erläuterungen zu den Anforderungen sowie um ein Reifegradmodell mit den Reifegraden , $0 “$ bis , ,3“ ergänzt. Hierbei galt die Einstufung „, 0 “ als unbewusster oder nicht vorhandener Teil des ITSM, wohingegen „,1“ das ad hoc oder initiale Bewusstsein über den Bestandteil des ITSM bedeutete. Der Reifegrad „2“ klassifizierte das Verständnis darüber, Aufgaben des ITSM wiederholbar, jedoch nicht in einem formal festgelegten Prozess durchzuführen. Der höchste Reifegrad „3“ stellte die optimale Erfassung von ITSM-Bestandteilen in definierter, dokumentierter und vollständiger Form dar. Es handelte sich bei dem Evaluierungsinstrument um ein Excel-basiertes Tool, das durch den Anwender einfach zu benutzen ist und eine effiziente Auswertung gewährleistet. Exemplarisch sind in Abb. 3 das Evaluierungsinstrument mit den ausgeklappten Informationstexten für GR1 und PR1 abgebildet.

Die Phase 2 fokussierte sich auf die Erhebung des aktuellen Stands des ITSM an den bayerischen HRZ. Hierfür wurden qualitative Daten von neun Hochschulen sowie deren Rechenzentren erhoben. In bilateralen Interviews von im Durchschnitt 60 min Länge wurden das Vorgehen und die Ziele des Projekts erläutert, das Evaluierungsinstrument vorgestellt sowie offene Fragen geklärt. In diesen Interviews wurde sich auch ein Überblick über die bereits vorhandene Kenntnisse zum Thema ITSM verschafft. Von Seiten der HRZ nahmen im Durchschnitt zwei Interviewpartner teil, die/der Leitende des HRZ und der/dir Serviceverantwortliche. Im Anschluss daran wurden die teilnehmenden HRZ gebeten, eine mit Hilfe des Evaluierungsinstruments geleitete Selbstbewertung ihres Standes des ITSM durchzuführen. Die Selbstbewertung wurde je nach Größe und Kapazität des HRZ mit variabler Dauer in Eigenregie durchgeführt. Die Rückmeldungen der teilnehmenden HRZ ergab eine durchschnittliche Bearbeitungszeit von etwa 90 min. Als Ergänzung und zusätzliche Informationsquelle für die Analyse diente auch eine Online-Lernplattform mit Erklärungsvideos, die ebenfalls durch die Autoren erarbeitet wurde. Die OnlineLernplattform zielt darauf $\mathrm{ab}$, detaillierte Informationen bezüglich der einzelnen Anforderungen bereitzustellen und diese detailliert zu erklären. Aufgrund der pandemischen Lage in den Jahren 2020 und 2021 war es nicht möglich, Workshops in Präsenz durchzuführen und Inhalte direkt zu vermitteln.

Nach Erhalt der ausgefüllten Evaluierungsinstrumente wurden in Phase 3 die Ergebnisse der Datenerhebung anonymisiert ausgewertet und verglichen. Die Antworten der ausgefüllten Evaluierungsinstrumente sind in Tab. 1 tabellarisch und anonymisiert aufgeführt. In Abschn. 4.1 sind die Antworten zweier Hochschulen in Form von Netzdiagrammen exemplarisch dargestellt, um die Unterschiede beim Stand der Hochschulen grafisch zu visualisieren und Schlüsselherausforderungen zu identifizieren. Des Weiteren wurden die Mittelwerte der einzelnen HRZ sowie je Anforderung berechnet, um auch diese Werte für die Analyse zu verwenden. 
Tab. 1 Übersicht der Datenerhebung der einzelnen HRZ

\begin{tabular}{llllllllll}
\hline $\begin{array}{l}\text { Anforde- } \\
\text { rungen }\end{array}$ & $\begin{array}{l}\text { Daten- } \\
\text { reihe } \\
1\end{array}$ & $\begin{array}{l}\text { Daten- } \\
\text { reihe }\end{array}$ & $\begin{array}{l}\text { Daten- } \\
\text { reihe }\end{array}$ & 3 & $\begin{array}{l}\text { Daten- } \\
\text { reihe } \\
4\end{array}$ & $\begin{array}{l}\text { Daten- } \\
\text { reihe } \\
5\end{array}$ & $\begin{array}{l}\text { Daten- } \\
\text { reihe } \\
6\end{array}$ & $\begin{array}{l}\text { Daten- } \\
\text { reihe } \\
7\end{array}$ & $\begin{array}{l}\text { Daten- } \\
\text { reihe } \\
8\end{array}$ \\
\hline GR1 & 1 & 2 & 2 & 2 & 2 & 3 & 2 & 0 & $\begin{array}{l}\text { Daten- } \\
\text { reihe } \\
9\end{array}$ \\
GR2 & 1 & 2 & 1 & 2 & 1 & 3 & 2 & 1 & 2 \\
GR3 & 1 & 3 & 1 & 2 & 2 & 3 & 1 & 1 & 2 \\
GR4 & 1 & 2 & 0 & 2 & 2 & 3 & 2 & 1 & 2 \\
GR5 & 1 & 2 & 0 & 2 & 2 & 3 & 2 & 1 & 2 \\
GR6 & 1 & 2 & 0 & 2 & 1 & 3 & 0 & 1 & 2 \\
GR7 & 1 & 2 & 0 & 2 & 2 & 3 & 2 & 1 & 2 \\
PR1 & 2 & 2 & 1 & 3 & 3 & 3 & 2 & 2 & 2 \\
PR2 & 1 & 2 & 1 & 3 & 1 & 3 & 1 & 1 & 3 \\
PR3 & 0 & 1 & 0 & 2 & 0 & 2 & 0 & 0 & 2 \\
PR4 & 1 & 2 & 2 & 2 & 2 & 3 & 2 & 1 & 1 \\
PR5 & 1 & 2 & 2 & 2 & 2 & 3 & 1 & 1 & 2 \\
PR6 & 1 & 3 & 2 & 2 & 2 & 3 & 3 & 2 & 1 \\
PR7 & 1 & 2 & 0 & 2 & 2 & 3 & 2 & 1 & 2 \\
PR8 & 2 & 3 & 3 & 2 & 1 & 3 & 0 & 2 & 3 \\
PR9 & 2 & 3 & 3 & 2 & 3 & 3 & 1 & 2 & 3 \\
PR10 & 1 & 2 & 1 & 1 & 2 & 2 & 2 & 1 & 2 \\
PR11 & 1 & 2 & 2 & 2 & 2 & 3 & 0 & 2 & 1 \\
PR12 & 1 & 3 & 0 & 2 & 2 & 3 & 2 & 1 & 2 \\
PR13 & 1 & 2 & 2 & 2 & 2 & 3 & 1 & 1 & 2 \\
PR14 & 1 & 1 & 1 & 1 & 1 & 3 & 2 & 1 & 2 \\
\hline & & & & & & & & &
\end{tabular}

Im Vordergrund der Phase 4 stand die Ableitung von Handlungsempfehlungen auf Basis der Ergebnisse der vorherigen Phase. Zusätzlich zu den Ergebnissen aus dem Evaluierungsinstrument wurden die Inhalte aus den Interviews in die Analyse mit einbezogen. Es wurden vier übergreifende Implikationen identifiziert, die folgend in Abschn. 4.2 vorgestellt werden. Unter anderem werden auf Basis der Handlungsempfehlungen im weiteren Verlauf des Projekts individuelle Empfehlungen für beteiligte Hochschulen ausgesprochen.

\section{Ergebnisse}

Im Folgenden werden die Lösungsansätze für die zuvor vorgestellten Phasen dargelegt. Die Datenerhebung des Status Quo des ITSM der bayerischen HRZ wurde in Form einer standardisiert geleiteten Selbstbewertung durchgeführt. Auf der Grundlage dieser Erhebung erfolgt die Analyse des aktuellen Stands des ITSM an den bayerischen HRZ (Abschn. 4.1) sowie das Erstellen und Ableiten von Handlungsempfehlungen (Abschn. 4.2). 


\subsection{Status Quo Analyse}

Da zunächst eine initiale Auswertung des Status Quo einer bayerischen Hochschule als Testlauf durchgeführt wurde, um mögliche Unstimmigkeiten im Rahmen der Datenerhebung zu vermeiden und dadurch eine einheitliche Datenerhebung zu gewährleisten, wurden im Anschluss daran, zwei Datensätze in einer vereinheitlichten Form eines Netzdiagramms übereinandergelegt, um zu analysieren, welche grundlegenden und spezifischen Anforderungen vergleichsweise besonders weit entwickelt sind und welche Anforderungen stärkeren Entwicklungsbedarf vorweisen. Abb. 4 stellt die Datensätze der Hochschulen 1 und 2 dar und gibt dabei einen Eindruck über die Antworten der HRZ.

Im Zuge dieser Analyse zeigt sich, dass sich die verschiedenen Hochschulen in unterschiedlichen Stadien befinden. Diesen Unterschied illustriert folgendes Beispiel:

Hochschule 2 befindet sich weitestgehend im Bereich des Reifegrads 2 (Mittelwert: 2,190), während sich Hochschule 1 überwiegend im Bereich des Reifegrads 1 (Mittelwert: 1,095) befindet.

Demzufolge bestehen im Bereich des ITSM innerhalb der unterschiedlichen HRZ Entwicklungsdiskrepanzen. Im Vergleich aller erhoben Datenreihen gilt jedoch flächendeckend, dass gerade die Bereiche des Information Security Managements (PR6) (Mittelwert: 2,111), des Service Portfolio Management (PR1) (Mittelwert:

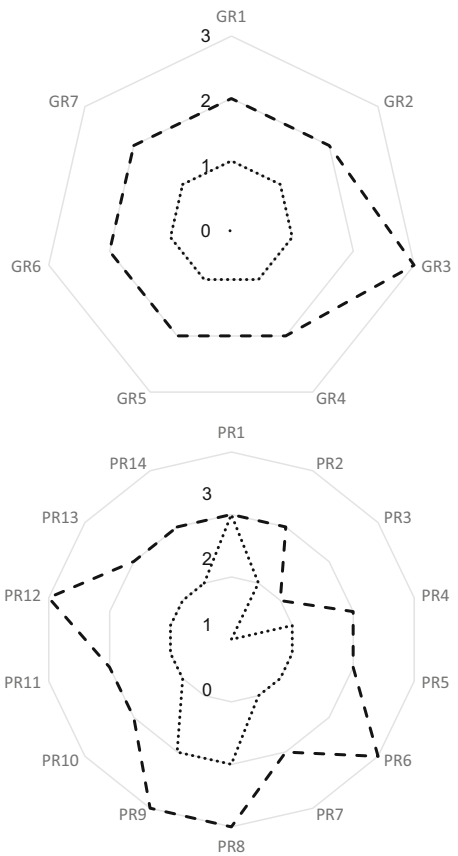
Legende
Hochschule 1
- - - - Hochschule 2
GR 1: Verpflichtung des Topmanagements
GR 2: Art und Umfang der Dokumentation
GR 3: Definition des Anwendungsbereiches
GR 4: PLAN: Planung
GR 5: DO: Implementierung
GR 6: CHECK: Überwachen und Überprüfen
GR 7: ACT: Kontinuierliche Verbesserung
PR 1: Service Portfolio Management
PR 2: Service Level Management
PR 3: Service Reporting Management
PR 4: Service Availability \& Continuity Management
PR 5: Capacity Management
PR 6: Information Security Management
PR 7: Customer Relationship Management
PR 8: Supplier Relationship Management
PR 9: Incident and Service Request Management
PR 10: Problem Management
PR 11: Configuration Management
PR 12: Change Management
PR 13: Release \& Deployment Management
PR 14: Continual Service Improvement Management

Abb. 4 Beispielhafte Übersicht der Erhebungsergebnisse 
2,222) und des Incident und Service Request Managements (PR9) (Mittelwert: 2,444) die durchschnittlich höchsten Reifegrade besitzen. Insbesondere in Zeiten der Digitalisierung von Services, beispielsweise des Lehrangebots, ist der Bereich des Information Security Management (PR 6) im Zuge der Corona-Pandemie essenziell geworden, um als HRZ widerstandsfähig gegenüber zunehmenden IT-Angriffen zu sein. Darüber hinaus wurde mittels der bilateralen Interviews zu Beginn der Datenerhebung sowie im Rahmen eines ergänzenden FitSM-Workshops mit FitSMExperte Dierk Söllner bereits deutlich, dass übergeordnet der Support seitens des Topmanagements essenziell für die operative und konkrete Umsetzung von ITSM ist. Ohne entsprechenden Topmanagement-Support fällt es den HRZ schwer, in die konkrete Umsetzung und Verbesserung von Prozessen einzusteigen. Im Gegensatz dazu scheinen die weiteren spezifischen Anforderungen unterschiedlich stark ausgeprägt zu sein. Demzufolge haben die HRZ unterschiedliche Prioritäten bezüglich der Bereitstellung ihrer IT Services. Diese unterschiedlichen Prioritäten gilt es individuell zu fördern, um einen einheitlichen Reifegrad zu erreichen und alle Aspekte des ITSM ganzheitlich zu erfassen.

Zusammenfassend lässt sich feststellen, dass ITSM in den beteiligten HRZ unterschiedlich stark etabliert ist. Dabei ist auch die Betrachtungsweise wichtig zu berücksichtigen, dass nicht jedes HRZ eine Zertifizierung nach ISO/IEC 20000 anstrebt beziehungsweise anstreben sollte. Viel eher muss berücksichtigt werden, welcher Reifegrad für die einzelnen HRZ im speziellen Kontext sinnvoll und umsetzbar ist. Eine Vorgehensweise, die wir weiterverfolgen, ist die Definition von Zielreifegraden für HRZ. Hierdurch können die HRZ beim jeweiligen Zielreifegrad so spezifisch wie möglich unterstützt werden.

\subsection{Allgemeine Erkenntnisse und Handlungsempfehlungen}

Im Anschluss an unsere Interviews und der Auswertung der Evaluierungen haben wir allgemeine Handlungsempfehlungen für ITSM von HRZ abgeleitet.

Empfehlung 1 Eine ITSM-Strategie für das jeweilige HRZ muss ausgehend von unterschiedlichen Reifegradstufen individualisiert gestaltet werden.

Durch die Analyse und Evaluierung der einzelnen HRZ hat sich ergeben, dass das Thema ITSM unterschiedlich stark in den jeweiligen HRZ verankert ist. Zum einen gibt es HRZ, die sich im Rahmen von IT-Sicherheitsthemen auch gleichzeitig mit ITSM auseinandergesetzt haben und somit erste Grundpfeiler für ITSMStrukturen gelegt wurden, zum anderen gibt es auch HRZ, die sich mit dem ITSM noch gar nicht beziehungsweise sehr wenig auseinandersetzt haben und wodurch noch keine Strukturen vorhanden sind. Dies lässt sich auch aus dem Vergleich der einzelnen Antworten der HRZ ableiten wie beispielsweise der Vergleich der Reifegrade von Hochschule 1 (Mittelwert: 1,095) und Hochschule 2 (Mittelwert: 2,190) zeigt. Für eine mögliche Vorgehensweise bedeutet dies, dass man kein pauschales Integrationskonzept zur Vereinheitlichung des hochschuleigenen ITSM aller HRZ entwickeln kann, sondern je nach Reifegrad individuell eine Strategie entwickeln muss. Beispielsweise kann ein HRZ, welches sich auf einem niedrigeren Reifegrad 
einordnet, mit der Erstellung eines Serviceportfolios beginnen, um initial den Status Quo und den Umfang des aktuellen Service Angebots abzustecken. Im Kontrast dazu identifiziert ein HRZ, welches sich tendenziell bei den höheren Reifegraden einordnet, konkrete Punkte zur Umsetzung einer professionellen (ITSM-)Zertifizierung (vgl. Abschn. 2).

\section{Empfehlung 2 Eine Involvierung der Hochschulleitung ist unabdingbar.}

Gerade durch die individuelle Selbstevaluierung der einzelnen HRZ ließ sich feststellen, dass das Thema ITSM durch einen ,top-down“-Ansatz geprägt ist. Zum einen konnte im Rahmen der Interviews festgestellt werden, dass das Thema ITSM vom Topmanagement beziehungsweise im Hochschulkontext, der Hochschulleitung, getrieben wird, um ITSM fokussiert anzugehen. Zum anderen ließ sich über die Länge des Bearbeitungszeitraums zur Beantwortung der Fragestellungen des Evaluierungsinstruments, zwischen drei Werktagen bis über 60 Werktage, feststellen, dass eine Motivation und eine Verpflichtung der Hochschulleitung für das Thema unabdingbar sind. Dies ist auch dadurch zu begründen, dass für eine Integration des ITSM in bestehende HRZ sowohl finanzielle als auch organisatorische Mittel notwendig sind, sei es über Prozesse, welche zu definieren, oder durch Stellen für Mitarbeitende, welche speziell für ITSM geschaffen und integriert werden müssen. Des Weiteren liegt das Thema ITSM nicht allein bei einem HRZ, sondern ist auch übergreifend für andere Abteilungen, zum Beispiel der Verwaltung, der Lehrstühle, der Bibliothekdienste, relevant und muss auch bei diesen Organisationseinheiten, welche nicht mehr nur dem HRZ zugeordnet sind, in gewissem Maße integriert und umgesetzt werden, wofür eine Unterstützung der Hochschulleitung unabdingbar ist.

\section{Empfehlung 3 Betrachtung der eigenen Leistungen als Service-Portfolio.}

Ein Aspekt, der im Rahmen der Evaluierung der HRZ aufgefallen und auch Teil der Anforderungen von FitSM, ITIL ${ }^{\circledR}$ und ISO/IEC 20000 ist, ist das Service-Portfolio. Basierend auf der Datenerhebung konnte die Erstellung einer trennscharfen Definition der angebotenen Services im Rahmen eines Service-Portfolios als essenzieller Teilaspekt eines professionellen ITSM abgeleitet werden. Dies bietet eine effiziente Möglichkeit, sich einen Überblick über die eigenen Leistungen des HRZ zu verschaffen und darüber hinaus auch die Verantwortlichkeiten des HRZ abzustecken. Dieser Überblick über die angebotenen Leistungen, ergänzt durch Informationen, wie zum Beispiel Verantwortung, Anlaufstelle, Verfügbarkeit und Umfang ergeben dann auch ein entsprechendes Service-Portfolio beziehungsweise Servicekataloge, welche unter anderem für eine ISO/IEC 20000 Zertifizierung gefordert sind (vgl. Abschn. 2). Die Erstellung ist deswegen ein fundamentaler Baustein zum Ausbau des ITSM, da basierend auf diesem Portfolio dann weitere Anforderungen, wie zum Beispiel Change-Management oder Service-Reporting, geprüft und integriert werden können. Dies zeigt auch unsere Evaluierung, wo die Anforderung Service Portfolio Management (PR1) mit einem Mittelwert von 2,222 zu denjenigen mit dem durchschnittlich höchsten Reifegrad zählt. 
Empfehlung 4 Die Thematik der IT-Sicherheit eignet sich als Einstieg für die Vertiefung des ITSM.

Im Rahmen der Ergebnisanalyse unserer Datenerhebungen mit den einzelnen HRZ hat sich ergeben, dass die Thematik der IT-Sicherheit ein sinnvoller Einstieg für die ITSM-Strategie bietet. Dies liegt unserer Meinung nach an zwei Aspekten. Zum einen ist das Thema IT-Sicherheit wie in Abschn. 1 beschrieben durch spezifische Auflagen in Deutschland auch in HRZ bereits rechtlich gefordert und deswegen schon sehr stark verbreitet. Dies spiegelt sich auch in den Ergebnissen der Datenerhebung der jeweiligen HRZ bei der Anforderung Information Security Management (PR 6) wider, welche im Vergleich zum allgemeinen Durchschnitt stärker ausgeprägt ist (Mittelwert: 2,111). Zum anderen können sich IT-Organisationen sowohl nach ISO/IEC 20000 (ITSM) als auch nach ISO/IEC 27000 (IT-Sicherheit) voneinander unabhängig zertifizieren lassen. Im Zuge dessen wird die Thematik der ITSicherheit häufig höher priorisiert, auch aufgrund gesetzlicher Anforderungen, und eignet sich daher als idealer Einstiegpunkt für einer Vertiefung des hochschuleigenen ITSM. Es kann in einem späteren Schritt für eine Zertifizierung nach ISO/IEC 20000 (ITSM) weiter ausgebaut werden. Wie in Abschn. 2 veranschaulicht, bieten ITSM und IT-Sicherheit aufgrund einer thematisch hohen Schnittmenge einen idealen Anknüpfungspunkt, um beginnend mit der IT-Sicherheit entsprechende Anforderungen gebündelt anzugehen.

\section{Fazit}

Um den aktuellen Stand von ITSM an bayerischen HRZ zu erfassen, wurden im Rahmen einer umfangreichen Fallstudie HRZ analysiert und auf Basis der Ergebnisse Handlungsempfehlungen herausgearbeitet. Dafür wurden zur Datenerhebung und Analyse neun HRZ interviewt und die Antworten des Evaluierungsinstruments ausgewertet. Hierbei ließ sich feststellen, dass der Reifegrad des ITSM bei den einzelnen HRZ unterschiedlich ausgeprägt und übergreifend kein klarer Trend zu erkennen ist. Im Rahmen unserer Handlungsempfehlungen haben wir deswegen auch unter anderem empfohlen, dass eine ITSM-Strategie für das jeweilige HRZ von sowohl der aktuellen Reifegradstufe als auch vom angestrebten Zielreifegrad individualisiert gestaltet werden muss. Eine Strategie die übergreifend für alle HRZ gilt, unabhängig vom bereits vorhandenen Reifegrad, ist daher nicht zu empfehlen.

Analog zu jeder Forschung ist dieser Beitrag nicht frei von Limitationen. Es sei darauf hingewiesen, dass die Ergebnisse und die daraus abgeleiteten Handlungsempfehlungen auf den Analysen von neun HRZ in Bayern bestehen. Um Aussagen auf nationaler oder gar internationaler Ebene zu treffen, müsste die Fallstudie ausgeweitet werden, um eine größere Datengrundlage zu erreichen.

Unabhängig von der Ausweitung der Fallstudie über ein größeres Feld von HRZ, kann der aktuelle Stand der Ergebnisse für eine Studie über die Entwicklung von HRZ genutzt werden, in dem die jeweiligen HRZ nach einiger Zeit noch ein weiteres Mal analysiert werden, um die Veränderung des Reifegrades zu beurteilen und damit Entwicklungstrends aufzuzeigen. Des Weiteren sollen die Ergebnisse des Projekts 
nun dafür verwendet werden, um individuell mit den beteiligten HRZ Konzepte auszuarbeiten, um deren ITSM zu verbessern, mit dem langfristigen Ziel, das ITSM an HRZ in Bayern und auch deutschlandweit weiter auszubauen.

Danksagung Die Autoren bedanken sich herzlich beim Staatsministerium für Wissenschaft und Kunst (StMWK) für die finanzielle Unterstützung als Fördergeber des Forschungsprojekts „IT-Servicemanagement für bayerische Hochschulrechenzentren “. Des Weiteren gilt der Dank den beteiligten Hochschulen beziehungsweise HRZ, die an der Datenerhebung, Analyse und Evaluierung beteiligt waren, welche im Sinne der Anonymität hier nicht namentlich aufgeführt werden können.

Funding Open Access funding enabled and organized by Projekt DEAL.

Open Access Dieser Artikel wird unter der Creative Commons Namensnennung 4.0 International Lizenz veröffentlicht, welche die Nutzung, Vervielfältigung, Bearbeitung, Verbreitung und Wiedergabe in jeglichem Medium und Format erlaubt, sofern Sie den/die ursprünglichen Autor(en) und die Quelle ordnungsgemäß nennen, einen Link zur Creative Commons Lizenz beifügen und angeben, ob Änderungen vorgenommen wurden.

Die in diesem Artikel enthaltenen Bilder und sonstiges Drittmaterial unterliegen ebenfalls der genannten Creative Commons Lizenz, sofern sich aus der Abbildungslegende nichts anderes ergibt. Sofern das betreffende Material nicht unter der genannten Creative Commons Lizenz steht und die betreffende Handlung nicht nach gesetzlichen Vorschriften erlaubt ist, ist für die oben aufgeführten Weiterverwendungen des Materials die Einwilligung des jeweiligen Rechteinhabers einzuholen.

Weitere Details zur Lizenz entnehmen Sie bitte der Lizenzinformation auf http://creativecommons.org/ licenses/by/4.0/deed.de.

\section{Literatur}

Abolhassan F (Hrsg) (2017) The drivers of digital transformation. Springer, Cham, S 1-10 https://doi.org/ 10.1007/978-3-319-31824-0

Beims M, Ziegenbein M (2021) IT-Service-Management in der Praxis mit ITIL; Zusammenarbeit systematisieren und relevante Ergebnisse erzielen. Hanser, München

Bick M, Börgmann K (2008) IT-Servicemanagement in deutschen Hochschulen. HMD 45:105-113. https://doi.org/10.1007/bf03341217

Bon (2008) Foundations of IT service management based on ITIL Bd. 3. Van Haren, Zaltbommel

Bosse E, Lübcke M, Book A, Würmseer G (2020) Corona@Hochschule - Befragung von Hochschulleitungen zur (digitalen) Lehre. HIS-Institut für Hochschulentwicklung e. V.

Fähnrich K-P, Bogdan F (2010) Informatik 2010; Service science - Neue Perspektiven für Informatik. Leipzig, 27.09.-01.10.2010. Bd. 1. Gesellschaft für Informatik, Bonn

FitSM (2016) FitSM-0 Overview and vocabulary. https://www.fitsm.eu/download/280/. Zugegriffen: 4. Juni 2021

Gaulke M (2019) Praxiswissen COBIT : Grundlagen und praktische Anwendung in der UnternehmensIT. Geeignet als Vorbereitung auf die ISACA-Prüfungen: COBIT Foundation, IT-Governance \& ITCompliance Practitioner, IT-Governance-Manager, IT-Compliance-Manager, CGEIT. dpunkt.verlag,

Hochschulrektorenkonferenz (2018) Informationssicherheit als strategische Aufgabe der Hochschulleitung. https://www.hrk.de/fileadmin/redaktion/hrk/02-Dokumente/02-04-Lehre/02-04-03-NeueMedien/HRK_MV_Empfehlung_Informationssicherheit_06112018.pdf. Zugegriffen: 4. Juni 2021

Hotzel H, Wimmer M, von der Heyde MLU (2015) IT Governance-role of a CIO in German Universities-a Survey by ZKI. Prax Informationsverarbeitung Kommunikation. https://doi.org/10.1515/pik2015-0019

Iden J, Eikebrokk TR (2013) Implementing IT service management: a systematic literature review. Int J Inf Manage 33:512-523. https://doi.org/10.1016/j.ijinfomgt.2013.01.004

Lörz M, Marczuk A, Zimmer L, Multrus F, Buchholz S (2020) Studieren unter Corona - Bedingungen: Studierende bewerten das erste Digitalsemester DZHW Brief 5. https://www.e-teaching.org/materialien/ literatur/loerz-marczuk-zimmer-multrus-buchholz-2020. Zugegriffen: 4. Juni 2021 
Rohrer A, Söllner D (2017) IT-Service-Management mit FitSM; Ein praxisorientiertes und leichtgewichtiges Framework für die IT. dpunkt.verlag, Heidelberg

Rudnicka J (2020) Anzahl der Hochschulen in Deutschland in den Wintersemestern 2015/2016 bis 2019/2020 nach Hochschulart. Statista. https://de.statista.com/statistik/daten/studie/247238/umfrage/ hochschulen-in-deutschland-nach-hochschulart/\#professional. Zugegriffen: 4. Juni 2021

Tiemeyer E (Hrsg) (2020) Handbuch IT-Management; Konzepte, Methoden, Lösungen und Arbeitshilfen für die Praxis. Hanser, München

Williamson B, Eynon R, Potter J (2020) Pandemic politics, pedagogies and practices: digital technologies and distance education during the coronavirus emergency. Learn Media Technol 45:107-114. https:// doi.org/10.1080/17439884.2020.1761641

Zhen W, Xin-yu Z (2007) An ITIL-based IT service management model for Chinese universities. In: Kim H-K (Hrsg) Fifth International Conference on Software Engineering Research, Management and Applications. SERA 2007 : proceedings Haeundae Grand Hotel, Busan, 20-22 August, 2007 IEEE Computer Society, Los Alamitos (in conjunction with 1st International Workshop on Advanced Internet Technology and Applications (AITA2007))

Zwilling M, Klien G, Lesjak D, Wiechetek Ł, Cetin F, Basim HN (2020) Cyber security awareness, knowledge and behavior: a comparative study. J Comput Inf Syst. https://doi.org/10.1080/08874417.2020. 1712269 